\title{
Erratum: Pump-Enhanced Continuous-Wave Magnetometry Using Nitrogen-Vacancy Ensembles [Phys. Rev. Applied 8, 034001 (2017)]
}

\author{
Sepehr Ahmadi, Haitham A.R. El-Ella, Jørn B. Hansen, Alexander Huck, and Ulrik L. Andersen \\ Department of Physics, Technical University of Denmark, 2800 Kongens Lyngby, Denmark
}

(Received 29 October 2018; published 26 November 2018)

DOI: 10.1103/PhysRevApplied.10.059901

We have identified an error in the calculation of the noise spectral density in the original article, which led to an incorrect spectrum plotted in Fig. 5. This led to an incorrectly estimated magnetic noise floor of $200 \mathrm{pT} / \sqrt{\mathrm{Hz}}$, and the incorrect conclusion of the measured noise floor being near-shot-noise limited. All other conclusions in the original article remain unaffected.

We corrected the error, as shown in Fig. 1, using Welch's method and a Blackman-Harris window function for estimation of the noise spectral density. From this plot, it is clear that the magnetic noise floor is approximately $2.5 \mathrm{nT} / \sqrt{\mathrm{Hz}}$. This value closely matches the directly extracted sensitivity discussed in the original article, and thus there is no discrepancy between these two estimation methods. In light of this correction, we now find that the magnetic noise floor is limited by the technical noise of the laser, which may be removed using a common-mode rejection scheme.
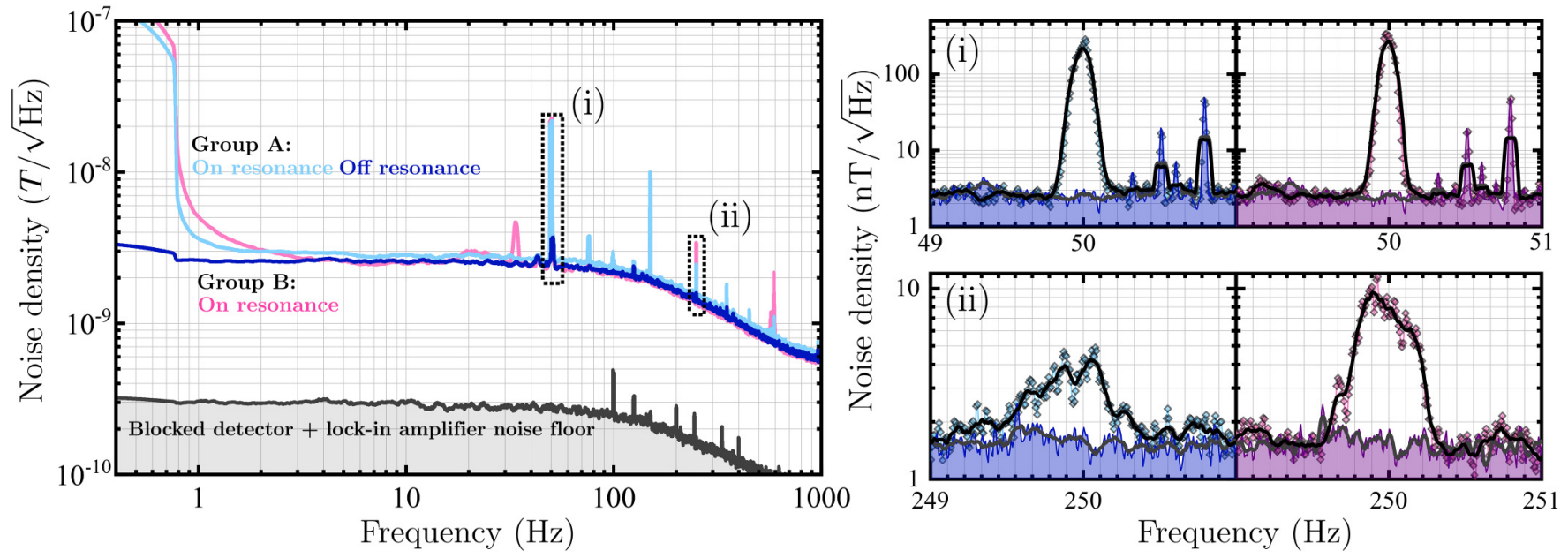

FIG. 1. The magnetic noise spectral density when on resonance $\left(\omega_{c}=\omega_{0}\right)$ and off resonance $\left(\omega_{c} \gg \omega_{0}\right)$ for groups A and B with $\Omega \sim 0.55 \mathrm{MHz}$ and $\Gamma_{p} \sim 0.3 \mathrm{MHz}\left(\mathcal{P}_{\text {in }} \sim 0.4 \mathrm{~W}\right)$. The combined noise floor of the lock-in amplifier and detector for the lowest gain settings of the lock-in amplifier, assuming $\max \left\{d_{\omega} \mathcal{S}_{\mathrm{LI}}\right\}$ similar to that of group A, is also plotted. The plots are averaged from five subsequently measured frequency traces and smoothed using a Savitzky-Golay filter. Magnified plots of the peaks designated (i) and (ii) show unsmoothed data points with the smoothed traces, highlighting the detection and lack of detection of the 50-Hz magnetic hum and its second odd harmonic when on or off resonance. The difference in amplitude is attributed to varying laboratory conditions.

*haitham.el@fysik.dtu.dk 